\title{
A PERCOLATION APPROACH TO STRONGLY INTERACTING MATTER
}

\author{
T. ÇELIK, F. KARSCH and H. SATZ \\ Department of Theoretical Physics, University of Bielefeld, Germany
}

Received 5 September 1980

\begin{abstract}
Using percolation theory to determine transition points, we show that strongly interacting bulk systems exhibit hadronic matter behaviour for densities $0.48 n_{0} \leqslant n \leqslant 14.0 n_{0}$ and quark matter behavior for $n \geqslant 3.84 n_{0}$, where $n_{0}=0.17 \mathrm{fm}^{-3}$ is nuclear density. For $3.84 n_{0} \leqslant n \leqslant 14.0 n_{0}$, we find a coexistence region of the two phases.
\end{abstract}

Possible phase transitions of strongly interacting matter from a hadron to a quark state have in the past years received considerable attention [1]. Recently Baym [2] suggested that this problem might be treated by percolation theory [3] and obtained some first estimates for "percolative" transition points. The aim of this note is to extend his considerations, showing that a percolation approach can provide both an appealing qualitative picture of the transitions in strongly interacting matter and rather reasonable quantitative values for the transition densities.

Hadrons are on one hand extended objects, on the other built-up of strongly confined quark constituents. The interaction between quarks must provide the scale for both the size of hadrons and for the range of hadronic forces as seen e.g. in typical ("soft" or "small" $p_{\mathrm{T}}$ ") scattering and production experiments [4]. Let us use this to determine some hadronic size parameters. For simplicity, we assume quarks inside a hadron (e.g., the $\mathrm{q}-\mathrm{q}$ pair in a pion) as confined in an infinite squarewell potential of radius $R_{\mathrm{Q}}$. If the same range also holds for the "soft" interaction force between to hadrons, then the radius of a hadron as seen in a scattering experiment is $2 R_{\mathrm{Q}}$; at a separation distance $r$ $\leqslant 2 R_{\mathrm{Q}}$, the quarks in each of the collision partners can be close enough to interact; for $r>2 R_{\mathrm{Q}}$, this is impossible (see fig. 1). Hence the volume of a hadron is given by $V_{\mathrm{H}}=8 V_{\mathrm{Q}}$, if $V_{\mathrm{Q}}=4 \pi R{ }_{\mathrm{Q}}^{3} / 3$ is the size of the confinement sphere or hadronic "core".

We can now easily distinguish different density regions. For a many hadron system of density $n \equiv N / V$



Fig. 1. Hadronic collision.

$\ll 1 / V_{\mathrm{H}}$, we have a hadron gas with interactions only during collisions - so we expect at equilibrium a free gas of hadrons. However, when the density becomes high enough to have arbitrarily large subsystems of clusters of hadrons in simultaneous interaction, then we shall speak of hadronic matter. Although hadronic matter thus provides a connected multihadron system, the quarks involved are in general still associated to a given hadron, as illustrated in fig. 2: all but one of the quarks shown are at distances $r>2 R_{\mathrm{Q}}$ from the antiquark $\mathrm{x}$. We are therefore not yet in a density regime corresponding to "delocalized" [2] quarks; this is reached only when the hadronic cores $V_{\mathrm{Q}}$ themselves form an interconnected cluster, since with overlapping

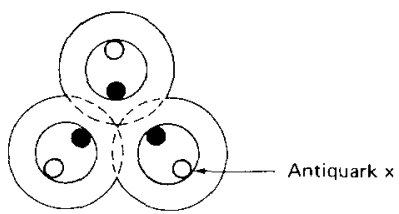

Fig. 2. Hadronic matter. 




Fig. 3. Quark matter.

cores (see fig. 3) one can no longer connect quarks to specific hadrons. In this case, we speak of quark matter. Summarizing, we distinguish three density regines: a free hadron gas if there is on the average no overlap of hadronic volumes, hadronic matter if in the thermodynamic limit there are infinite clusters of overlapping hadrons, but not of their cores, and finally quark matter if there are infinite core clusters.

Let us now recall the basic question asked by percolation theory [3]. If we put conducting spheres into an arbitrarily large box, allowing overlap, at what density of spheres will current be able to flow ? Numerical studies [5] indicate that this "percolation" will occur when the fraction 0.28 of (three-dimensional) space is covered by spheres, or - equivalently - when the sum of the sphere volumes makes up $34 \%$ of space.

Since the onset of current flow also signals the onset of infinite cluster formation, the connection between percolation and the different density patterns of strongly interacting matter becomes evident. We thus find the beginning of the hadronic matter region at the percolation density

$n_{\mathrm{H}}=0.34 / V_{\mathrm{H}}$,

for hadron size spheres. With a hadronic radius $\left(R_{\mathrm{H}}\right.$ $=2 R_{\mathrm{Q}}$ of $1 \mathrm{fm} V_{\mathrm{H}}^{-1}=0.24 \mathrm{fm}^{-3}$, so that we expect hadronic matter formation at densities

$n \geqslant n_{\mathrm{H}}=0.48 n_{0}$

where $n_{0}=0.17 \mathrm{fm}^{-3}$ is nuclear density. Similarly, core percolation starts at

$n_{\mathrm{Q}}=0.34 / V_{\mathrm{Q}}=2.72 / V_{\mathrm{H}}$,

so that for densities

$n \geqslant n_{\mathrm{Q}}=3.84 n_{0}$,

we expect quark matter formation.

It appears physically reasonable that in both cases there will be a coexistence regime beyond the percolation point. Thus, for $n<n_{\mathrm{H}}$, we have only a hadron gas, but slightly above $n=n_{\mathrm{H}}$ both hadronic matter and hadron gas; similarly, just above $n=n_{\mathrm{Q}}$ we expect both hadronic and quark matter. To obtain estimates for the sizes of these coexistence regions, we may consider "inverse" of "vacuum bubble" percolation. Reducing the density of hadronic or quark matter will eventually lead to the formation of "matterless" bubbles of a certain size, and we may ask at what density these will form an infinite connected cluster.

If we fill a box of size $V$ with $N$ spheres of size $V_{\mathrm{H}}$, allowing overlap, then

$\left[\left(V-V_{\mathrm{H}}\right) / V\right]^{N}$

is the fractional volume not covered by spheres. At the percolation point for hadronic matter, $29 \%$ of space is covered, so that

$$
\left[\left(V-V_{\mathrm{H}}\right) / V\right]^{N} \approx 0.71
$$

is the fraction remaining empty. Writing for large $V$

$$
\begin{aligned}
(1 & \left.-V_{\mathrm{H}} / V\right)^{N} \approx \exp \left[-(N / V) V_{\mathrm{H}}\right] \\
& =\exp \left(-n_{\mathrm{H}} V_{\mathrm{H}}\right)=0.71,
\end{aligned}
$$

we recover the percolation density (1). Applying the same argument to the formation of vacuum bubbles of size $V_{\mathrm{H}}$, we obtain

$$
\left[\left(V-V_{\mathrm{H}}\right) / V\right]^{N} \approx 0.29 \text {, }
$$

for the fraction of space covered by the vacuum bubble percolation point. This yields, arguing as in eq. (7)

$\bar{n}_{\mathrm{H}} \approx 1.24 / V_{\mathrm{H}}=1.75 n_{0}$,

as the highest density at which the vacuum can still form an infinite cluster of size $V_{\mathrm{H}}$ bubbles. - Similarly, $V_{\mathrm{Q}}$ or core-sized vacuum bubble percolation ceases at

$\bar{n}_{\mathrm{Q}}=1.24 / V_{\mathrm{Q}}=9.92 / V_{\mathrm{H}}=14.0 n_{0}$.

We thus obtain the picture shown in fig. 4 for the transition of strongly interacting matter from hadron gas to quark matter.

In closing we note that for a hard sphere hadron gas [6], solidification sets in at 0.74 of close-packing density. With the parameters chosen here, this yields.

$n_{\mathrm{c}}^{\mathrm{H}}=0.77 n_{0}$,

as the critical density for the phase transition from hadron gas to hadron solid. For a gas of quarks confined in square-well potentials [7] the phase transition from 


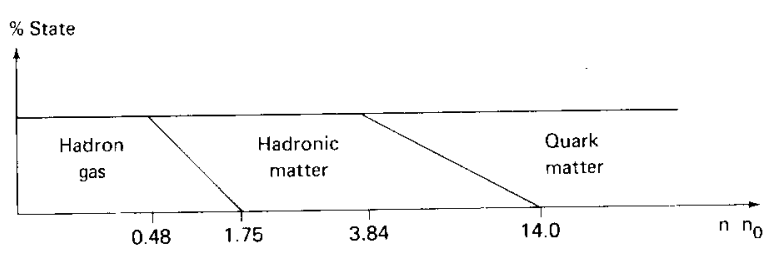

Fig. 4. Strongly interacting matter as a function of density; $n_{0}=$ nuclear density.

bound to unbound constituents occurs at $0.4 / V_{\mathrm{Q}}$; with $V_{\mathrm{H}}=8 V_{\mathrm{Q}}$ this implies

$n_{\mathrm{c}}^{\mathrm{Q}}=4.5 n_{0}$,

for the critical density, above which the quarks can move freely within matter. The results of the percolation picture thus agree rather well with other models also constructed to provide a single description of strongly interacting matter on both sides of the transition point.

\section{References}

[1] For recent reviews, see e.g.:

G. Baym, in: Nuclear physics with heavy ions and mesons, Les Houches Lectures 1977 (North-Holland, Amsterdam, 1978);

D.J. Gross, R.D. Pisarski and L.G. Yaffe, QCD and instantons at finite temperature, preprint (1980); M. Kislinger and P. Morley, Phys. Rep. 51 (1979) 64; E.V. Shuryak, Phys. Rep. 61 (1980) 72.

[2] G. Baym, Physica 96A (1979) 131

[3] For recent reviews, see e.g.:

S. Kirkpatrick, Rev. Mod. Phys. 45 (1973) 574;

B. Payandeh, Nuovo Cimento 3 (1980) 1;

D. Stauffer, Phys. Rep. 54 (1979) 1.

[4] H. Satz, Nuovo Cimento 37A (1977) 141.

[5] V.K.S. Shante and S. Kirkpatrick, Adv. Phys. 20 (1971) 325.

[6] F. Karsch and H. Satz, Phys. Rev. D21 (1980) 1168.

[7] F. Karsch and H. Satz, Phys. Rev. D22 (1980) 480. 